\title{
Open-charm production measurements with ALICE at the LHC
}

\author{
P. Pagano ${ }^{1,2, a}$ on behalf of the ALICE Collaboration \\ ${ }^{1}$ Salerno University - Italy \\ 2I.N.F.N. - Gruppo Collegato di Salerno
}

\begin{abstract}
The LHC heavy-ion physics program aims at investigating the properties of strongly-interacting matter under extreme conditions of temperature and energy density where the formation of the Quark-Gluon Plasma (QGP) is expected. Heavy-flavour hadrons, containing charm and beauty quarks, are considered efficient probes to investigate the properties of the QGP produced in heavy-ion collisions. Heavy quarks are produced in hard partonic scattering processes in the initial stage of hadronic collisions and propagate through the hot and dense medium created in the collision losing energy interacting with the medium via radiative and collisional processes. The high precision tracking, good vertexing capabilities and excellent particle identification offered by the ALICE experiment allow us to measure particles containing heavy quarks in a wide transversemomentum range in $\mathrm{pp}, \mathrm{p}-\mathrm{Pb}$ and $\mathrm{Pb}-\mathrm{Pb}$ collisions. A review of the main results on prompt $\mathrm{D}$-mesons production, reconstructed via their hadronic decays at mid-rapidity, in pp collisions at $\sqrt{s}=7 \mathrm{TeV}, \mathrm{p}-\mathrm{Pb}$ collisions at $\sqrt{s_{N N}}=5.02 \mathrm{TeV}$ and $\mathrm{Pb}-\mathrm{Pb}$ collisions at $\sqrt{s_{N N}}=2.76 \mathrm{TeV}$ will be shown.
\end{abstract}

\section{Introduction}

The ALICE experiment at the LHC aims to investigate the properties of the high energy-density and strongly-interacting matter created in heavy-ion collisions at ultrarelativistic energies. Charm and beauty quarks are an excellent probe to study it. Due to their large masses, they are produced predominantly in hard parton scattering processes at the early stages of the collisions. Therefore, they pass through all the phases of the system evolution losing energy in their interactions with other medium constituents. The flavour is conserved in these interactions so heavy quarks can be considered sensitive probes of the medium properties. Open heavy-flavour hadron production is expected to be sensitive to the energy density of the system through the mechanism of in-medium energy loss of heavy quarks [1-3]. Quantum Chromodynamics (QCD) theoretical calculations predict a dependence of the energy loss on the mass of the parton traversing the medium, according to the dead cone effect [4], which suppresses the induced gluon radiation at small angles for heavy partons. Furthermore, the energy loss through the medium depends on the parton colour charge and path length. This results in an expected hierarchy of the parton energy loss, with beauty quarks losing less energy than charm quarks, and charm quarks losing less energy than light quarks and gluons. One of the observables that are sensitive to the interaction of hard partons with the medium is the nuclear modification factor, $R_{A A}$,

ae-mail: paola.pagano@sa.infn.it 
defined as the ratio of the particle yield measured in $\mathrm{Pb}-\mathrm{Pb}$ collisions and the yield in pp collisions scaled by the number of binary collisions. $R_{A A}$ is expected to be equal to one for heavy flavours in the absence of medium effects. The expected hierarchy of the energy loss described above can be probed comparing the $R_{A A}$ of different particle species, respectively, B, D mesons and light-hadrons [5]. Further knowledge of the properties of the medium created in heavy-ion collisions can be gained from the study of the azimuthal anisotropy of open heavy flavours: the initial spatial asymmetry is transformed into an asymmetry in momentum via hydrodynamic expansion of the medium. This is quantified in terms of the second harmonic coefficient $v_{2}$ of the Fourier expansion of the D-meson azimuthal distribution. The elliptic flow parameter $v_{2}$ brings information on the medium transport properties, in particular on the question whether heavy quarks take part in the collective expansion of the medium and on the path-length dependence of energy loss.

The interpretation of particle production measurements in $\mathrm{Pb}-\mathrm{Pb}$ collisions requires detailed studies and understanding of their production in $\mathrm{pp}$ and $\mathrm{p}-\mathrm{Pb}$ collisions. $\mathrm{pp}$ collisions provide the essential reference for the nuclear modification factor and a sensitive test of perturbative QCD models. In addition, with pp collisions it is possible to gain information on the role of Multiple-Parton Interactions (MPI), i.e. several hard partonic interactions occurring in a single collision between two nucleons. Measurements of open heavy-flavour in $\mathrm{p}-\mathrm{Pb}$ collisions allow to disentangle cold nuclear matter (CNM) effects (such as transverse momentum broadening, gluon saturation at low Bjorken-X [6], modification of the nuclear parton distribution functions [7, 8], energy loss [9]) from final-state effects that occur in a deconfined medium.

\section{ALICE detector layout and open heavy-flavour reconstruction}

The ALICE detector [10] is well suited for heavy-flavour measurements at the LHC in a wide momentum and rapidity range. The detectors in the central barrel used for this analysis are: the Inner Tracking System (ITS), the Time Projection Chamber (TPC) and the Time-of-Flight detector (TOF). The ITS comprises six layers of silicon pixel, strip and drift detectors and is used for the tracking of charged particles and the reconstruction of the primary and secondary vertex. Track reconstruction in the central barrel $(|\eta|<9)$ starts from the TPC, which also provides particle identification (PID) by measuring the specific energy loss $\mathrm{d} E / \mathrm{d} x$ of charged particles passing throught it. Further PID information is given by the TOF via the time stamp of the charged particle hits on the detector with respect to the time of the interaction. The cooperation of these high performing detectors allows the excellent performance needed for heavy-flavour analyses. Moreover the V0 scintillator detectors at forward and backward rapidities provide minimum bias trigger and are used to determine the centrality of $\mathrm{Pb}-\mathrm{Pb}$ collisions.

Prompt D mesons are fully reconstructed through their hadronic decay channels: $D^{0} \rightarrow K^{-} \pi^{+}$, $D^{+} \rightarrow K^{-} \pi^{+} \pi^{+}, D^{*+} \rightarrow D^{0} \pi^{+}, D_{s}^{+} \rightarrow \phi \pi^{+} \rightarrow K^{+} K^{-} \pi^{+}$and their charge conjugates. The reconstruction is based on the selection of their decay secondary vertex, displaced by few hundred $\mu \mathrm{m}$ from the primary vertex and topological selections, combined with particle identification criteria, applied to reduce the high combinatorial background.

The signal yield is obtained by fitting the invariant mass distributions of the candidates, while the contribution from beauty feed-down is estimated based on FONLL pQCD calculations and subtracted in order to evaluate the results for prompt D meson yield [11].

The D-meson yield is measured as a function of the charged-particle multiplicity in pp and p$\mathrm{Pb}$ collisions. The $\mathrm{D}$ meson yields and the charged-particle multiplicity are both normalized to the multiplicity-integrated values. In the case of $\mathrm{p}-\mathrm{Pb}$ collisions, the nuclear modification factor $R_{p P b}$ is defined as the $\mathrm{D}$-meson cross section in $\mathrm{p}-\mathrm{Pb}$ collisions divided by the measured cross section in $\mathrm{pp}$ collisions scaled by the lead mass number. 
The $p_{T}$-differential cross section measurements of prompt $\mathrm{D}$ meson have been performed in $\mathrm{pp}$ collisions at $\sqrt{s}=2.76 \mathrm{TeV}$ [12] and $\sqrt{s}=7 \mathrm{TeV}$ [13] and the results are well described by perturbative QCD. Because of the limited statistics of the $\sqrt{s}=2.76 \mathrm{TeV}$ sample, a $\sqrt{s}$ extrapolation is applied to define the pp reference needed for the $R_{A A}$ calculation.

The prompt D-meson elliptic-flow parameter $v_{2}$ is also reported as a function of $p_{T}$ in semiperipheral collisions (30-50\% centrality class).

\section{Results}

The $p_{T}$-differential cross sections for $D^{0}, D^{+}, D^{*+}$ and $D_{s}^{+}$at $\sqrt{s}=7 \mathrm{TeV}$ are shown in Fig. 1, together with FONLL and GM-VFNS model calculations. Within uncertainties, theoretical predictions and measurements are in agreement. Nevertheless, it can be noted that the measurements tend to be higher than the central value of the FONLL prediction; for GM-VFNS, instead, the data lie on the lower side of the predictions.
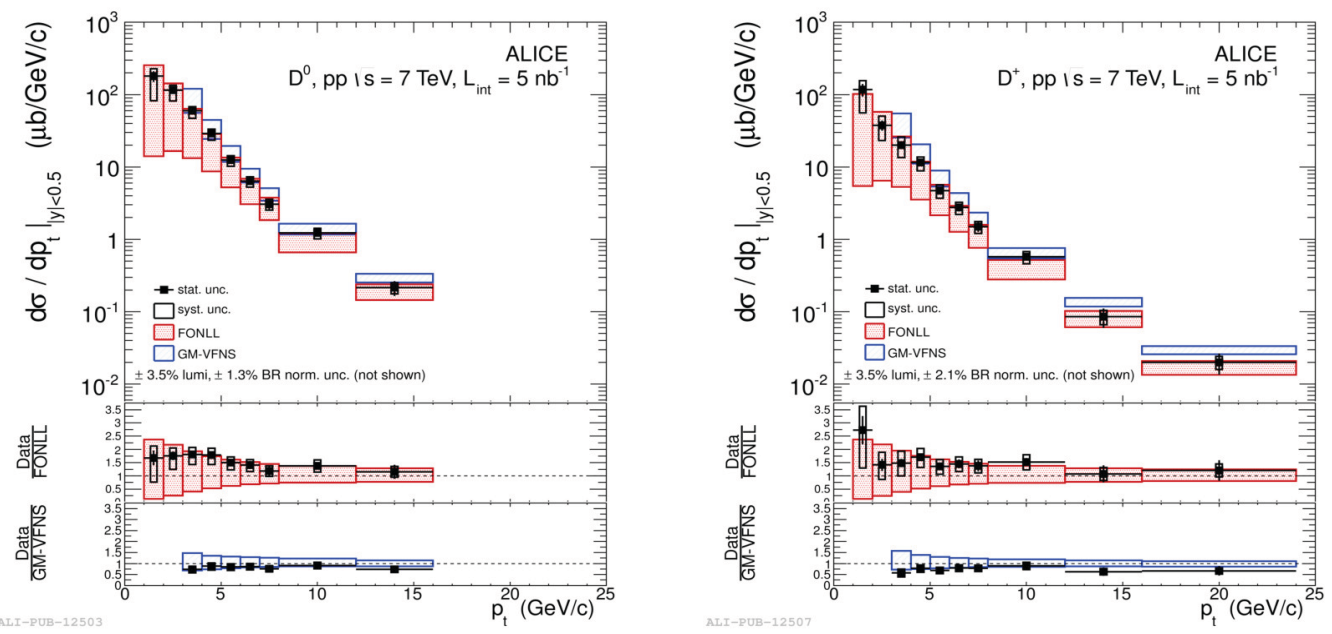

Figure 1. $p_{T}$-differential inclusive cross section for prompt $D^{0}$ and $D^{+}[12]$ mesons in pp collisions at $\sqrt{s}=7 \mathrm{TeV}$ compared with FONLL [7] and GM-VFNS [15] theoretical predictions. The symbols are positioned horizontally at the centre of each $p_{T}$ interval.

In the left panel of Fig. 2 the average of $D^{0}, D^{+}$and $D^{*+}$ self-normalized yield as a function of relative charged- particle multiplicity is shown for $\mathrm{pp}$ and $\mathrm{p}-\mathrm{Pb}$ collisions in the transverse-momentum range $2<p_{T}<4 \mathrm{GeV} / c$. The result for pp collisions indicates that D-meson yields increase with the charged-particle multiplicity by a factor of about 15 in the range between 0.5 and 6 times $\left\langle\mathrm{d} N_{c h} / \mathrm{d} \eta\right\rangle$. The increase of the heavy-flavour self-normalized yields with the charged-particle multiplicity is qualitatively consistent with the theoretical calculations including the contribution from MPI to heavyflavor production at LHC energies [16]. A similar behaviour was observed also for $\mathrm{p}-\mathrm{Pb}$ collisions. However, in this case, high-multiplicity events also come from the higher number of binary nucleonnucleon collisions.

In the right panel of Fig. 2 shows the average $R_{p P b}$ and $R_{A A}$ of prompt $D^{0}, D^{+}$and $D^{*+}$ mesons as a function of $p_{T}[18,19]$. The $R_{p P b}$ of D mesons is consistent with unity for $p_{T}>2 \mathrm{GeV} / c$, and is described by calculations that include CNM effects [20-24]. 

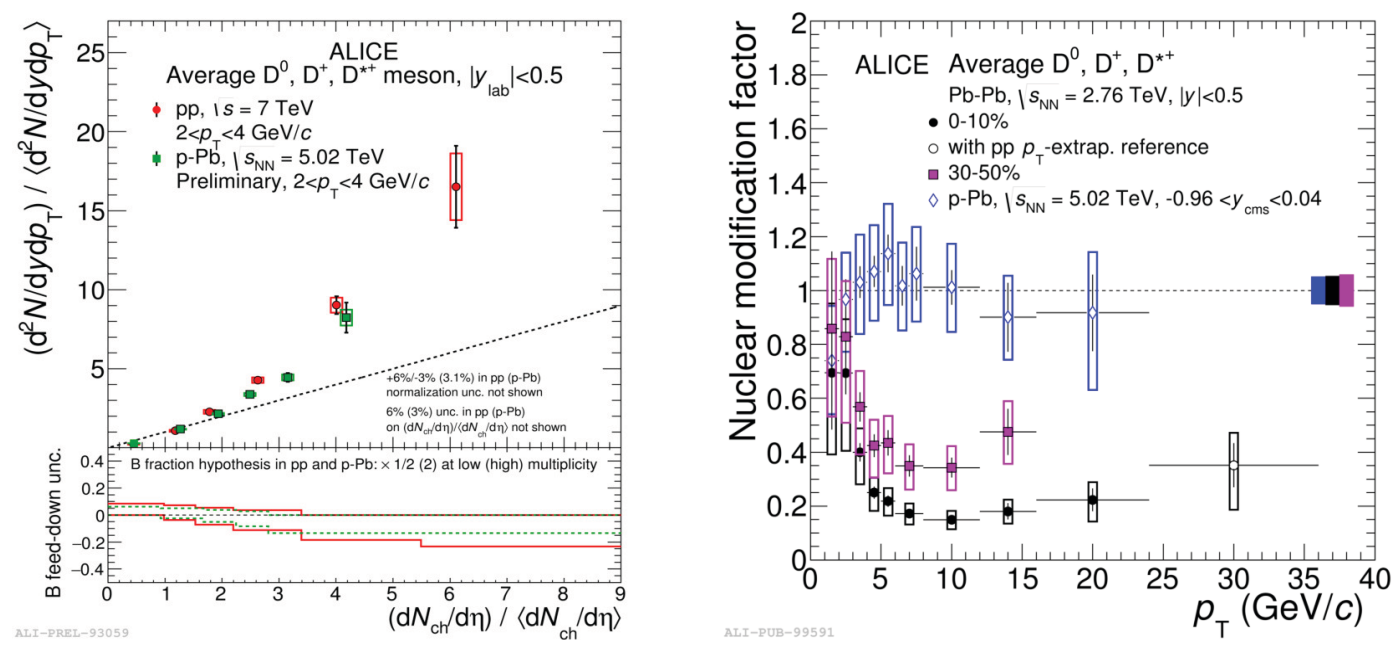

Figure 2. Left: Average D-meson yields as a function of charged-particle multiplicity in pp at $\sqrt{s}=7 \mathrm{TeV}$ [17] and $\mathrm{p}-\mathrm{Pb}$ at $\sqrt{s_{N N}}=5.02 \mathrm{TeV}$ for $2<p_{T}<4 \mathrm{GeV} / c$. D mesons are reconstructed in $\left|y_{\text {lab }}\right|<0.5$. The results are presented in the top panels with their statistical (vertical bars) and systematic (boxes) uncertainties, except for the feed-down fraction uncertainty that is drawn separately in the bottom panels. Right: Prompt D-meson nuclear modification factor (average of $D^{0}, D^{+}$and $D^{*+}$ ) as a function of $p_{T}$ in $\mathrm{Pb}-\mathrm{Pb}$ collisions at $\sqrt{s_{N N}}=2.76 \mathrm{TeV}$ in the $0-10 \%$ and $30-50 \%$ centrality classes [19] superimposed with the same measurement in $\mathrm{p}-\mathrm{Pb}$ collisions at $\sqrt{s_{N N}}=5.02 \mathrm{TeV}[18]$.

The nuclear modification factor in $\mathrm{Pb}-\mathrm{Pb}$ collisions, $R_{A A}$, shows a significant suppression for $p_{T}$ $>3 \mathrm{GeV} / \mathrm{c}$ in both the $0-10 \%$ and $30-50 \%$ centrality classes; in particular a larger suppression is observed for the most central collisions, as expected. As the $R_{p P b}$ remains consistent with unity over the entire measured $p_{T}$ range, it is concluded that the suppression in $\mathrm{Pb}-\mathrm{Pb}$ collisions can be attributed to final-state effects in the medium rather than CNM effects.

In the left panel of Fig. 3 the D-meson $R_{A A}$, that it is the average of $D^{0}, D^{+}$and $D^{*+}$, as a function of the centrality of $\mathrm{Pb}-\mathrm{Pb}$ collisions, expressed as the average number of nucleons that participate in the collision $N_{\text {part }}$, is shown for $8<p_{T}<16 \mathrm{GeV} / c$ [25]. The centrality dependence of D-meson $R_{A A}$ was studied in the range between 0 and $80 \%$. In the same figure the D-meson $R_{A A}$ is compared to the non-prompt $\mathrm{J} / \Psi R_{A A}$ coming from beauty-hadron decays measured by the CMS Collaboration [26]. The chosen $p_{T}$ ranges imply that both $\mathrm{D}$ and $\mathrm{B}$ mesons probed by these measurement have average $p_{T}$ of about $10 \mathrm{GeV} / c$ in a slightly different y range. A suppression that increases towards central collisions was observed for both D mesons and non-prompt J/ $\Psi R_{A A}$. The measurements show a Dmeson $R_{A A}$ lower than the non-prompt $\mathrm{J} / \Psi R_{A A}$ in central collisions, suggesting a larger energy loss for charm than for beauty quarks, as expected from theoretical calculations that predict a mass-dependent energy loss. The predictions from a model [27], including mass-dependent energy loss for charm and beauty quarks, are superimposed to the measurements: the D-meson and non-prompt $\mathrm{J} / \Psi R_{A A}$ can be well described by the this calculation.

Figure 3 (right) shows the comparison of the D-meson $R_{A A}$ with that of charged hadrons and pions as a function of $p_{T}$. The measurements show a similar suppression from mid to high $p_{T}$ but this is still consistent with models including parton charge and mass dependence of energy loss. 

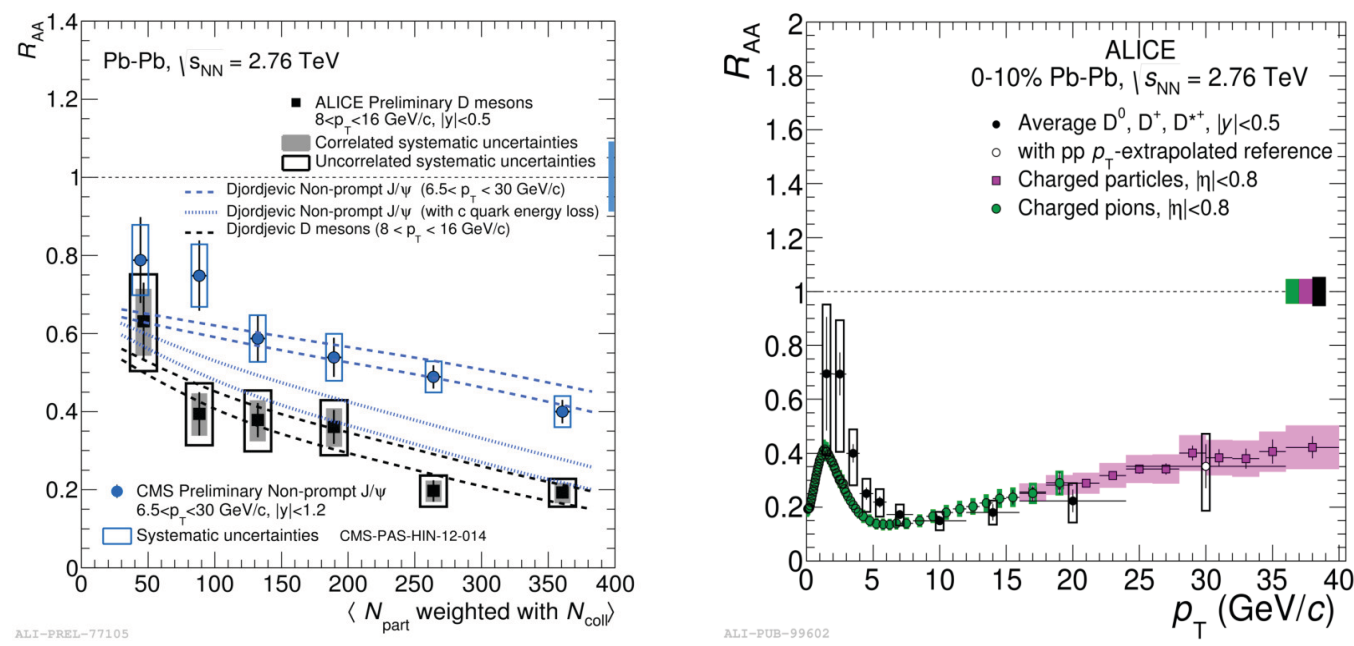

Figure 3. Left: Average $D^{0}, D^{+}$and $D^{*+} R_{A A}$ vs $N_{\text {part }}$ for the $8<p_{T}<16 \mathrm{GeV} / c$ (black points) [25]. Non-prompt $\mathrm{J} / \psi R_{A A}$ vs $N_{\text {part }}$ measured by CMS for $|\mathrm{y}|<1.2,6.5<p_{T}<30 \mathrm{GeV} / c$ (blue points) [26]. The data points are compared to Djordjevic models for D mesons (dashed black line), non-prompt $\mathrm{J} / \psi$ (dashed blue line) and also considering the charm mass for the non-prompt $\mathrm{J} / \psi$ (dotted blue line). Right: Average of prompt $D^{0}, D^{+}$and $D^{*+}$ $R_{A A}$ in the centrality class $0-10 \%$ compared to pions and charged particles.

The average $v_{2}$ of the three $\mathrm{D}$-meson species measured in 30-50 \% central $\mathrm{Pb}-\mathrm{Pb}$ collisions, shown in the right panel of Fig. 4, is larger than zero with a $5.7 \sigma$ significance in the interval $2<p_{T}<6 \mathrm{GeV} / c$. This result indicate that at low $p_{T}$ charm quarks participate in the collective motion of the system. A postive $v_{2}$ is also observed for $p_{T}>6 \mathrm{GeV} / c$ but for a more qualitative study of the path-length dependence of parton energy loss via $v_{2}$ measurements more statistics is needed.

In both panels of Fig. 4 [36] the ALICE measurements are compared to various theoretical calculations including in-medium parton energy loss [28-35]. Most models describe qualitatively well the prompt D-meson $R_{A A}$ as a function of $p_{T}$, in particular the models including both collisional and radiative energy loss. The low $p_{T} v_{2}$ result is best described by models that include the collective expansion of the medium and a mechanism of hadronisation via recombination of charm quarks with light quarks. Nevertheless, the simultaneous description of the large suppression of D mesons in central collisions and their anisotropy in non-central collisions is challenging for theoretical models.

\section{Conclusions}

The ALICE experiment at the LHC has an excellent performance for heavy-flavor measurements, thanks to tracking, vertexing and PID. Results on the D-meson production measurements from ALICE were reported in $\mathrm{pp}, \mathrm{p}$ - $\mathrm{Pb}$ and $\mathrm{Pb}$ - $\mathrm{Pb}$ collisions. The $p_{T}$-differential cross sections for prompt $\mathrm{D}$ mesons in pp collisions are described within uncertainties by theoretical predictions based on $\mathrm{pQCD}$ calculations. The D-meson self-normalized yields exhibit an increasing trend with increasing chargedparticle multiplicity in pp collisions, suggesting that MPIs affect the hard momentum scale relevant for heavy-flavour production. A similar increase is observed in $\mathrm{p}-\mathrm{Pb}$ collisions, but in this case a higher number of binary nucleon-nucleon collisions also contributes to high-multiplicity events. The $R_{p P b}$ of prompt $\mathrm{D}$ mesons in $\mathrm{p}-\mathrm{Pb}$ collisions is consistent with unity within uncertainties, providing 

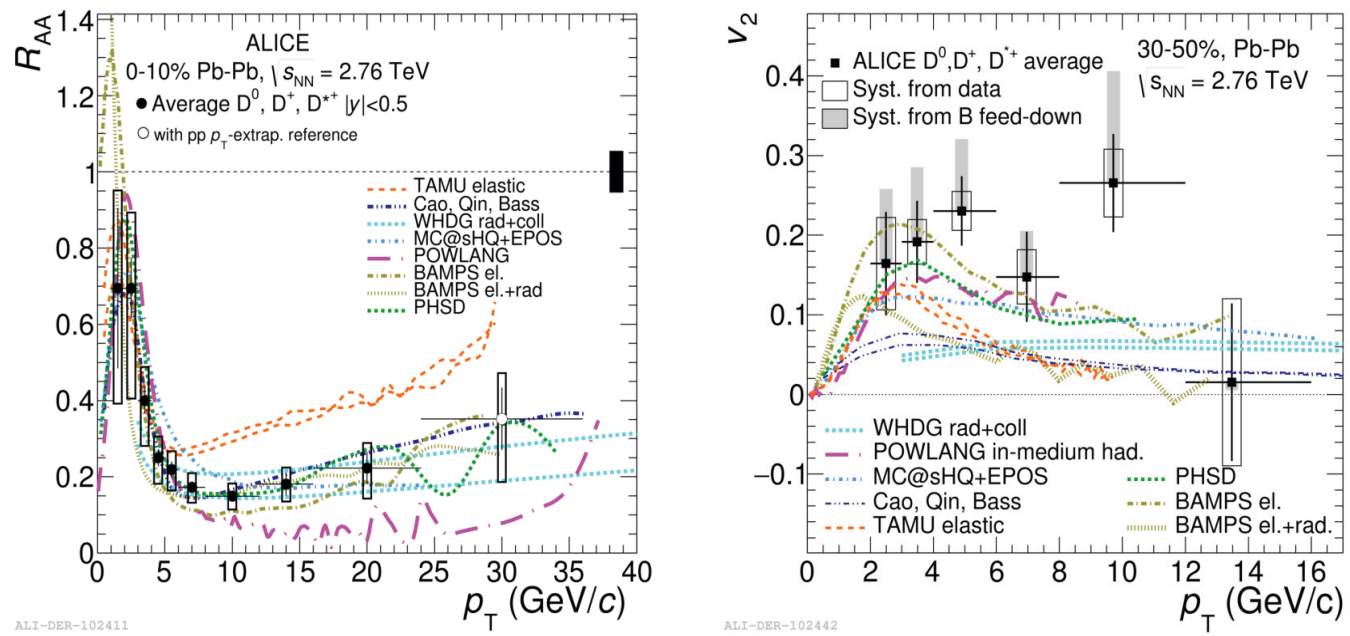

Figure 4. Left: Model comparisons for average D meson $R_{A A}$ in the $0-20 \%$ centrality class. Right: Model comparisons for average D meson $v_{2}$ in the $30-50 \%$ centrality class. In both panels the seven model calculations are: WHDG rad+coll [28], POWLANG [29], Cao, Qin, Bass [30], MC@sHQ+EPOS [31], BAMPS [32], TAMU elastic [33], UrQMD [34, 35]. The models WHDG rad+coll, POWLANG, TAMU elastic and UrQMD are shown by two lines that represent their uncertainty.

no indication for large $\mathrm{CNM}$ effects at high $p_{T}$. In the most centra $\mathrm{Pb}-\mathrm{Pb}$ collisions, a strong suppression of heavy-flavour yields is observed at intermediate and high $p_{T}$ with respect to binary-scaled pp collisions. As the influence of CNM effects is small in the measured $p_{T}$ range, these results in $\mathrm{Pb}-\mathrm{Pb}$ collisions can be interpreted as a final-state effect due to in-medium parton energy loss, consistent with the expected parton-mass dependence. The $v_{2}$ measured in $\mathrm{Pb}-\mathrm{Pb}$ semi-central collisions is larger than zero at low $p_{T}$, suggesting that heavy quarks participate in the collective motion of the system.

\section{References}

[1] M. Gyulassy and M. Plumer, Phys. Lett. B243 432 (1990).

[2] R. Baier, Y. L. Dokshitzer, A. H. Mueller, S. Peigne and D. Schiff, Nucl. Phys. B484 265 (1997).

[3] M. H. Thoma and M. Gyulassy, Nucl. Phys. B351 491 (1991).

[4] Y. L. Dokshitzer, D. E. Kharzeev, Phys. Lett. B519 (2001) 199.

[5] A. Adil, I.Vitev, Phys. Lett. B 649 (2007)139.

[6] H. Fujii, K. Watanabe, Nucl. Phys. A 915 (2013) 1.

[7] K. Eskola, H. Paukkunen and C. Salgado, JHEP 04 (2009) 065.

[8] D. de Florian and R. Sassot, Phys.Rev. D 69 (2004) 074028.

[9] B. B. Abelev et al., ALICE Collaboration, Int. J. Mod. Phys. A 29 (2014) 1430044.

[10] B. B. Abelev et al., ALICE Collaboration, Int. J. Mod. Phys. A 29 (2014) 1430044.

[11] M. Cacciari et al., CERN-PHTH 2011227.

[12] B. Abelev et al., ALICE Collaboration, JHEP 07 (2012) 191.

[13] B. Abelev et al., ALICE Collaboration, JHEP 01 (2012) 128. 
[14] B. Abelev et al., ALICE Collaboration, Phys. Lett. B 718 (2012) 279.

[15] G. Aad et al., ATLAS Collaboration, Eur.Phys. J. C 72 (2012).

[16] K. Aamodt et al., ALICE Collaboration, JINST 3 S08002 (2008).

[17] B. Abelev et al., ALICE Collaboration, JHEP 09 (2012) 148.

[18] B. Abelev et al., ALICE Collaboration, Phys. Rev. Lett. 113 (23) (2014) 232301.

[19] B. Abelev et al., ALICE Collaboration, Preprint (2015). arXiv:1509.06888.

[20] R. Sharma, I. Vitev, B.-W. Zhang, Phys. Rev. C 80 (2009) 054902.

[21] K. J. Eskola, H. Paukkunen, C. A. Salgado, JHEP 04 (2009) 065.

[22] M. L. Mangano, P. Nason, G. Ridolfi, Nucl. Phys. B 373 (1992) 295-345.

[23] D. Stump, et al., JHEP 10 (2003) 046.

[24] H. Fujii, K. Watanabe, Nucl. Phys. A 920 (2013) 78-93.

[25] B. Abelev et al., ALICE Collaboration, Preprint (2015). arXiv:1506.06604.

[26] V. Khachatryan et al., CMS Collaboration, CMS-PAS-HIIN-12-014.

[27] Djordjevic, Phys. Lett. B 734 (2014) 286-289.

[28] W.A. Horowitz, M. Gyulassy, Nucl Phys. A 872 (2011)265.

[29] M.Monteno et al., Jour. Phys. G 38 (2011) 124144.

[30] S. Cao, G. Qin, S.A. Bass, Phys.Rev. C 88 (2013) 044907.

[31] E. Retinskaya, M. Luzum, J. Ollitrault, Phys.Rev. C 89 (2014) 014902.

[32] J. Uphoffa, O. Fochlera, Z. Xub, C. Greinera, Phys.Lett. B 717 (2012) 430.

[33] He, Min et al., Phys.Rev. B 735 (2014) 445.

[34] T. Lang, H. van Hees, J. Steinheimer, M. Bleicher, Phys. Rev. C 93014901 (2016).

[35] T. Lang et al, J. Phys. Conf. Ser. 426012032 (2013).

[36] B. Abelev et al., ALICE Collaboration, Phys. Rev. C 90 (2014)034904. 